\title{
Linx
}

Revue des linguistes de l'université Paris X Nanterre

42 | 2000

Approches sociolinguistiques du plan phonique

\section{Affaiblissement consonantique en cadien}

\section{Chantal Lych}

\section{OpenEdition}

\section{Journals}

Édition électronique

URL : http://journals.openedition.org/linx/770

DOI : $10.4000 /$ linx.770

ISSN : 2118-9692

\section{Éditeur}

Presses universitaires de Paris Nanterre

\section{Édition imprimée}

Date de publication : 1 juin 2000

Pagination : 29-43

ISSN : 0246-8743

Référence électronique

Chantal Lych, «Affaiblissement consonantique en cadien », Linx [En ligne], 42 | 2000, mis en ligne le 21 juin 2012, consulté le 03 mai 2019. URL : http://journals.openedition.org/linx/770 ; DOI : 10.4000/ $\operatorname{linx} .770$

Ce document a été généré automatiquement le 3 mai 2019.

Département de Sciences du langage, Université Paris Ouest 


\title{
Affaiblissement consonantique en cadien
}

\author{
Chantal Lych
}

1 Les phénomènes de la parole rapide ont pendant longtemps été relégués à la périphérie de la phonologie et pourtant, comme le souligne Grammont (1933:170) «Dans toutes les langues, il se produit à certaines époques un relâchement de l'articulation, plus ou moins considérable, plus ou moins général. " Le français ne fait certainement pas exception à cette loi et toutes les variétés sont concernées même si la nature et l'ampleur de ces relâchements ne concordent pas d'une variété à l'autre. L'intérêt sera porté ici sur les chutes de segments, l'élision étant sans nul doute le degré ultime de l'affaiblissement. Il ne saurait être question ici de nouvelles tendances puisque certains effacements sont largement notés au seizième siècle, et que Palsgrave (cité dans Thurot, 1883 : 314) signale déjà qu'une coda simple ou complexe est muette à l'intérieur d'un mot. En français dit standard, ces relâchements sont considérés comme l'apanage d'un français familier, d'une langue non surveillée (Gadet, 1992, Laks, 1977, Wachs, 1997). Notre étude portera sur un ensemble de chutes de consonnes dans une variété 'avancée' de français, le cadien. ${ }^{1}$ Le cadien est une langue orale, non standardisée encore parlée par environ 200000 personnes dans l'Etat de Louisiane (Girard \& Lyche, 1998).

2 Nous nous proposons ici d'envisager deux registres distincts du cadien afin de déterminer la direction de cette simplification..$^{2}$ Le cadien étant jusqu'à ces dernières années une langue exclusivement orale, la langue écrite n'a jamais pu influer sur la prononciation et les chutes de consonnes sont en fait devenues 'la norme' (Conwell \& Juilland, 1963, Papen $\&$ Rottet, 1997). Nous comparerons les données de ces deux registres et nous ébaucherons une analyse dans le cadre de la théorie de l'optimalité qui renoue avec la tradition fonctionnaliste et redonne aux explications phonétiques toute leur force. Nous verrons comment les relâchements observés sont le résultat de l'interaction de contraintes universelles dont l'effet diffère dans des langues particulières. 


\section{Chute de consonnes en cadien}

3 Les enregistrements sur lesquels porte cette étude nous permettent de distinguer deux registres. Le premier correspond à un parler lent, un style soutenu, une situation assez formelle. Il s'agit d'une entrevue avec un homme d'une soixantaine d'années, maire d'une petite ville au nord de Lafayette. Ce locuteur (désormais 'A') nous a reçues dans son bureau de maire et nous a présenté sa ville, la situation linguistique et les actions que sa municipalité avaient entreprises pour sauvegarder la langue et la culture cadiennes. Le français cadien était sa langue maternelle mais il ne le lisait ni l'écrivait, toute sa scolarité ayant été faite en langue anglaise. En tant qu'enquêteurs nous n'avons pas tenté délibérément de provoquer une discussion plus naturelle et nous avons conduit cet entretien dans un style que l'on pourrait qualifier de causerie journalistique. L'autre registre est plus naturel, les discussions sont beaucoup plus spontanées. Il s'agit soit d'une locutrice interviewée sur fond de musique cadienne, soit d'enregistrements effectués par des Cadiens dans un contexte familial, soit enfin d'histoires racontées par plusieurs personnes lors d'émissions radiophoniques. Tous ces enregistrements sont ponctués de rires, les thèmes de discussion ne présentent pas de véritable continuité et les répétitions sont fréquentes. On retrouve en un mot tous les éléments qui caractérisent la conversation spontanée. ${ }^{3}$

4 Tout d'abord, nous éliminerons de l'ensemble de nos données un certain nombre de formes lexicalisées, les deux premières étant d'ailleurs en passe de le devenir aussi en français dit standard.

\begin{tabular}{|c|c|c|}
\hline (1) & parce que & [paskø] \\
\hline & plus & [py] \\
\hline & manière & [maje:r] \\
\hline & autres & {$[\mathrm{ot}]$} \\
\hline & juste & [3ys] \\
\hline
\end{tabular}

5 Le pronom tonique des deux premières personnes du pluriel est systématiquement renforcé par l'adjectif autres (nous autres, vous autres), mais la forme de autres est toujours privée de la liquide comme dans les aut' pays, les aut' parties. A ces quelques exemples, on peut ajouter quelqu'un prononcé soit [kckõ] ou [kikõ] ainsi que petit qui se présente le plus souvent sous sa forme réduite [pti] mais dont la forme accentuée est [piti].

\subsection{Registre formel}

6 Parmi les différents cas d'élision, le plus systématique affecte la liquide dans un groupe obstruante + liquide (OL) en finale de mot, presque indépendamment, peut-on dire, de l'environnement phonétique. 


\begin{tabular}{|c|c|c|}
\hline (2) & ça se fait battre et d'autres vient & [sasf\&batedotvj $\tilde{\varepsilon}]$ \\
\hline & dans septembre & [dãs\&ptãb] \\
\hline & quand quelqu'un jongle & [kãkعkœ̃̃zõg] \\
\hline & le maître de & [lømetdø] \\
\hline & si la lettre est trop long & [silalet\&trolõ] \\
\hline
\end{tabular}

7 Le groupe entier peut disparaître comme dans (3).

\begin{tabular}{|c|c|c|}
\hline (3) & un centre de pétrole & [œ̃sãdøpctro] \\
\hline & un cercle avec le bâton & [@̃sscraveløbatõ] \\
\hline
\end{tabular}

8 Sur la base de (2) et (3), on pourrait être tenté d'émettre l'hypothèse que les codas OL ont été relexicalisées en $\mathrm{O}$. Dans le parler de A cependant, la liquide du groupe OL peut faire surface en situation d'enchaînement et dans les dérivés.

\begin{tabular}{|l|l|l|}
\hline$(4)$ & comment ce miracle a été fait & [kJmãsømiraklaetef\&] \\
\hline & on va célébrer & [õvaselebre] \\
\hline \hline & de montrer à les petits & [dømõtrealepti] \\
\hline
\end{tabular}

9 La liquide peut même se manifester devant consonne, à l'intérieur d'un syntagme phonologique comme dans notre fête [notrãf $\varepsilon$ :t]. Un autre exemple est le chiffre quatre prononcé [kat] systématiquement sauf lorsqu'il se trouve être le premier élément du composé quatre-vingts [katrøvẽ]].

10 La réduction des groupes consonantiques ne se limite pas aux groupes obstruante + liquide, d'autres groupes sont aussi affectés.

\begin{tabular}{|l|l|l|}
\hline$(5)$ & pas juste préserver & [pa3ysprezerve] \\
\hline & ils gardent la langage & [iga:dlalãga:3] \\
\hline & le parc national & [løpa:knasjonal] \\
\hline
\end{tabular}

11 Comme nous le voyons en (5), il faut considérer plusieurs cas de figure : la dernière consonne d'un groupe tombe sauf s'il s'agit d'un groupe $r+C$, auquel cas c'est à la liquide de tomber. Dans le même enregistrement, nous avons aussi un exemple où un groupe /lt/ disparaît complètement : à faire la récolte [larekJ:]. La chute de /r/ devant consonne est 
aussi très courante à l'intérieur d'un mot et, lors de l'interview, notre locuteur a utilisé à plusieurs reprises et pour développer une idée qui lui semblait chère, la forme [pa:1],

\begin{tabular}{|l|l|l|}
\hline$(6)$ & si tu parles deux langues & [sitipa:ldø] \\
\hline & si tu parlais & [sitipa:lø] \\
\hline
\end{tabular}

alors que l'autre forme est largement présente ailleurs :

\begin{tabular}{|l|l|l|}
\hline (7) & le monde parle français & [lømõdparlfrãs $\varepsilon]$ \\
\hline \hline & et personne parle français & [epersonparlfrãs $]$ \\
\hline
\end{tabular}

13 (6) représente les seuls cas de chute de / $\mathrm{r} /$ interne chez A. Dans tous les autres mots, un / $\mathrm{r}$ / interne de morphème n'est jamais amuï (le dernier [lãdernje], personne [person]), même lorsqu'il précède un schwa non prononcé : dernièrement [dernjermã]. A ne simplifie pas maintenant [mẼtnã] alors que [mẼnnã] est très courant en cadien.

14 Les groupes consonantiques ne sont pas les seuls affectés, une consonne simple peut chuter en finale de mot et cette disparition semble liée à certains items lexicaux.

\begin{tabular}{|c|c|c|}
\hline (8) & avec le bâton & [aveløbatõ] \\
\hline & pour sûr & [pusyr] \\
\hline & maîtresses d'école ${ }^{4}$ & [metresdeko:] \\
\hline & le monde des musiciens & [lmõdemyzisyz̃ ] \\
\hline
\end{tabular}

15 Tous ces cas de relâchement mettent en cause la coda de la syllabe et plus particulièrement la coda de morphème. Nous avons en effet indiqué que les codas internes sont plus stables et dans l'ensemble les attaques ne sont pas concernées. Pour compléter ce tour d'horizon, ajoutons que dans cette interview, le locuteur utilise uniquement la forme réduite de je ([3]), qu'aucun schwa interne n'est prononcé dans le contexte VCschwa alors que les schwas rythmiques (les schwas de monosyllabes et les schwas de syllabe initiale de mot) peuvent être réalisés comme dans de ceux de la Louisiane ([døsødølalwizjan]). La première syllabe de l'imparfait de l'auxiliaire être est tronquée ( était [tع], tout le monde était comme ça [tylmõdtckomsa]), et le démonstratif féminin ne possède que la forme /st/ (cette année [stane]).

\subsection{Registre spontané}

16 Dès que la conversation devient plus informelle, les relâchements se multiplient mais ne sont cependant pas généralisés. La fréquence augmente et les contextes où la chute est possible s'élargissent. Dans les groupes OL en finale de mot, la chute de la liquide est généralisée qu'il y ait ou non possibilité d'enchaînement. ${ }^{5}$ 


\begin{tabular}{|c|c|c|}
\hline (9) & par exemple & [parعgzãp] \\
\hline & le pauvre nègre là & [løpovnegla] \\
\hline & la prendre elle & [laprãdcl] \\
\hline & mon sucre et ma crème & [mõsykemakrعm] \\
\hline
\end{tabular}

Le groupe tout entier est susceptible de tomber comme dans cinq mille piastres de fine [š̃kmilpjasdøfajn]. A ces chutes tout à fait systématiques s'ajoutent aussi de très nombreuses chutes de $/ \mathrm{r} /$ devant consonne en finale de mot.

\begin{tabular}{|c|c|c|}
\hline (10) & regarde & [rga:d] \\
\hline & mange des tartes & [mãzdeta:t] \\
\hline & tu fermais la porte & |[tifermelapo:t] \\
\hline & plus ta ferme était grand & 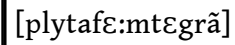 \\
\hline
\end{tabular}

18 A l'intérieur d'un mot, c'est-à-dire en position de coda simple, qu'il s'agisse d'une frontière morphologique ou non, la chute de $/ r$ / est aussi très fréquente.

\begin{tabular}{|c|c|c|}
\hline (11) & regardons celui-là & [rgadõsuila] \\
\hline & qui sortait à peu près & [kisつ:tEapãpre] \\
\hline & on est après chercher ${ }^{6}$ & 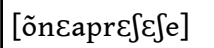 \\
\hline & personne & [peson] \\
\hline & comme aujourd'hui & [komozudui] \\
\hline
\end{tabular}

Pour donner une image complète de la situation en finale absolue, nous devons aussi envisager les groupes obstruante + obstruante. Nos données ne nous en fournissent que peu d'exemples. On relève évidemment juste prononcé systématiquement sans l'occlusive, de même la disparition $d u / t /$ dans reste, ou aussi celle de la fricative dans valse. Nous n'avons qu'un seul exemple d'occlusive + occlusive, il s'agit de correct et la deuxième occlusive tombe, ce qui donne [korek].

Avant d'aborder les consonnes finales simples, considérons brièvement le groupe OL à l'initiale de syllabe. A l'initiale de mot, le groupe OL est en position forte et la liquide devrait être stable. C'est d'ailleurs ce que l'on observe chez A. On sait cependant que dans les créoles du français la liquide peut tomber dans cette position et que cette faiblesse de $/ \mathrm{r} /$ peut être interprêtée comme la continuation d'une tendance qui n'a pas pu se développer en France à cause de la résistance de forces conservatrices (Tinelli, 1981 : 172). 
A ce sujet, notons que Wachs (1997) ne rapporte qu'un seul cas de chute dans un groupe initial dans son enquête sur le français de l'Ile de France. Ici, mis à part A, tous les locuteurs sont susceptibles d'omettre /r/ dans une attaque syllabique initiale de mot ou non.

\begin{tabular}{|c|c|c|}
\hline (12) & c'était un vaillant prêtre & [stعœ̃vajãp\&:t] \\
\hline & tu viens trop tard & [tivjẽtota:r] \\
\hline & nous montrer nos prières & [numõtrenopic:r] \\
\hline & il est après faire & [ilcapeff:r] \\
\hline
\end{tabular}

21 Ce phénomène demeure cependant rare et de faible ampleur, ce qui n'est pas le cas de la chute d'une consonne en finale de mot. Nous avons vu que chez A, une consonne finale était relativement stable. Dans le reste du corpus les chutes sont fréquentes et la consonne la plus faible dans cette position est sans nul doute $/ \mathrm{r} /$.

\begin{tabular}{|c|c|c|}
\hline (13) & j'aime ça noir & [3Emsanwa:] \\
\hline & pour fouiller ce jour là & [pufujes3u:la] \\
\hline & et dire nos prières & [edirnopric:] \\
\hline & pour mourir & [pumuri] \\
\hline & chaudière à laver & [SodjEalave] \\
\hline
\end{tabular}

Les occlusives sont beaucoup plus stables, mais au-delà de certains items tels avec, monde qui perdent fréquemment leur consonne, on remarque quelques chutes sporadiques.

\begin{tabular}{|l|l|l|}
\hline$(14)$ & mes jambes là & {$[\mathrm{m} \varepsilon 3 a ̃ l a]$} \\
\hline \hline & au ras des trois grosses buttes & [oradetrwagrosby] \\
\hline
\end{tabular}

Ces enregistrements témoignent aussi de nombreux cas d'élision de voyelle en début de mot au-delà de ce qui a été relevé pour $\mathrm{A}$. Ces élisions se produisent de préférence lorsque le mot précédent se termine par une voyelle phonétiquement identique.

\begin{tabular}{|l|l|l|}
\hline (15) & beaucoup contents ensemble & [bokukõtãsãb] \\
\hline & l'a acheté & [lafte] \\
\hline & s'il a pas arrangé ça & [silaparãzesa] \\
\hline
\end{tabular}


Enfin, tous les schwas internes et l'immense majorité des schwas rythmiques sont éliminés.

Une comparaison de la langue de A en situation d'interview assez formelle avec les autres enregistrements montre - en situation dite spontanée - que le cadien élimine totalement les groupes OL à la finale des mots et que les codas en /r/ ne sont pas stables (que la consonne soit l'unique constituent de la coda ou le premier élément d'une coda branchée).

La chute d'une consonne en position de coda n'est pas une spécificité cadienne, on la retrouve à plus ou moins grande échelle dans tous les dialectes du français. En acadien, (Motapanyane, 1997), les groupes OL sont simplifiés comme en cadien, dans les groupes $\mathrm{sCC}$, la dernière consonne tombe toujours et souvent seul le /s/ est prononcé (piastre [pjas]). Si le groupe final a la forme $\mathrm{sC}$, la dernière consonne tombe : est [عs], casque [kas]. Dans le Sud de la France, région pourtant réputée pour son maintien systématique de schwa, l'élision consonantique est néanmoins répandue. La liquide d'un groupe OL final est généralement protégée par un schwa, ce qui assure son maintien (membre de l'opposition), mais est instable dans le contexte OLschwa\#V. De même certains items lexicaux perdent leur occlusive finale et un groupe obstruante + obstruante peut être simplifié. ${ }^{7}$

\begin{tabular}{|l|l|l|}
\hline$(17)$ & la table était ouverte à tout le monde (PD) & {$[$ tab] } \\
\hline & des trucs comme ça & {$[$ try] } \\
\hline & à voir avec Bordeaux (MD) & {$[\mathrm{av} \varepsilon]$} \\
\hline
\end{tabular}

27 Ces tendances ont été observées de longue date et Brun (1931) mentionne la réduction des groupes consonantiques comme l'une des grandes caractéristiques du français de Marseille. Une occlusive en coda interne tombecomme dans affe'tion, o'server, au'menter, a'jectif, se'tembre, ce que Brun (1931 : 43) commente ainsi :

Ces réductions, déjà signalées dans Gabrielli, qui donne de longues listes, sont générales dans les classes populaires, extrêmement fréquentes encore dans la petite bourgeoisie.

L'intérêt de la situation en cadien réside moins dans les faits eux-mêmes (puisque nous savons que de tels relâchements ponctuent toutes les variétés de français) que dans leur amplitude et leur fréquence. Nous avons vu qu'ils touchaient tous les registres et une comparaison de ces registres nous permet d'établir une progression, d'envisager pleinement le développement du phénomène. Le contexte le plus susceptible d'être affecté par la chute de consonnes est principalement la finale absolue, une chute de consonne interne de mot et les réductions de voyelles à l'initiale sont plus inhibées lorsque le locuteur est en situation formelle.

\section{Relâchements et théorie de l'optimalité}

Tous les processus de relâchement se caractérisent par une minimisation de l'effort articulatoire (Passy, 1891, Grammont, 1933, Kirchner, 1998), certains gestes étant remplacés par d'autres dont le coût est moindre. Cette tendance universelle s'oppose 
néanmoins à une autre tendance qui veut que la communication se fasse dans les meilleures conditions possibles, que la compréhension soit maximale. La théorie de l'optimalité (Prince \& Smolensky, 1993) nous permet de formaliser ces observations, d'incorporer des explications 'phonétiques' dans l'analyse. Selon la théorie de l'optimalité (désormais OT), la grammaire universelle comprend un ensemble de contraintes qui, bien qu'universelles, ne sont pas respectées de la même façon dans toutes les langues. Les contraintes n'ont pas dans une langue donnée le même poids, elles sont rangées selon leur importance et leur hiérarchie varie de langue à langue. La phonologie d'une langue est alors réduite à une hiérarchie spécifique de ces contraintes. Nous allons maintenant tenter de définir les contraintes à l'oeuvre dans nos données, mais nous pouvons déjà affirmer que leurs effets sont conflictuels: d'un côté des contraintes de fidélité qui protègent les structures existantes en prônant l'inaction 'ne faites rien, ne touchez à rien' afin de maintenir une bonne compréhension, s'opposent à des contraintes de marque qui tendent vers des structures non marquées, des structures qui réduisent l'effort articulatoire. La contrainte de fidélité cruciale pour notre propos est MAXIO selon laquelle tous les éléments de la forme de base se retrouvent dans la forme de surface. L'effet de MAXIO est d'interdire l'élision.

\begin{tabular}{|l|l|ll|}
\hline (18) & MAXIO : & $\begin{array}{l}\text { Il existe une correspondance maximale entre la forme de base et la forme de } \\
\text { surface. }\end{array}$ \\
\hline
\end{tabular}

MAXIO se heurte aux forces innovatrices de la langue qui, comme le rappelle Kiparsky (1988: 377), sont de trois types: des processus prosodiques, des relâchements et des renforcements. Les renforcements se rencontrent de préférence en syllabe accentuée, alors que les relâchements ont lieu en position inaccentuée, affectent la coda de la syllabe et caractérisent un registre informel. Tous nos exemples concordent avec ces dernières observations que Venneman (1988:2) attribue au principe suivant :

Diachronic Maxim : Linguistic change on a given parameter does not affect a language structure as long as there exist structures in the language system that are less preferred in terms of the relevant preference law.

En d'autres termes, les structures qui sont tout d'abord touchées par un changement pour un paramètre donné sont les structures les plus 'faibles', les structures défavorisées par des lois générales. Dans le cas qui nous concerne, la majorité des segments affectés sont des consonnes de coda. Le cadien, comme bien d'autres langues, préfère la syllabe canonique CV (Lyche, 1997) définie par trois contraintes (Prince \& Smolensky, 1993) :

\begin{tabular}{|l|l|l|}
\hline$(19)$ & ATTAQUE : & Une syllabe doit contenir une attaque \\
\hline & *CODA : & Une syllabe ne contient pas de coda \\
\hline & CODA-COND : & Si une syllabe contient une coda, celle-ci doit être liée à l'attaque suivante. \\
\hline
\end{tabular}

Reprenons tout d'abord quelques principes qui régissent la structure des codas dans les langues qui les autorisent. Venneman (1988: 21) résume un ensemble d'observations dans une loi de préférence, la loi des codas. 
Coda Law : A syllable coda is the more preferred: (a) the smaller the number of speech sounds in the coda, (b) the less the Consonant Strength of its offset, and (c) the more sharply the Consonant Strength drops from the offset toward the Consonantal Strength of the preceding syllable nucleus.

5 L'échelle de force consonantique qu'il propose est identique à l'échelle de sonorité :

occlusives sourdes $>$ occlusives sonores $>$ fricatives sourdes $>$ fricatives sonores $>$ nasales $>$ liquides $(/ \mathrm{l} / \mathrm{)})>$ liquides $(/ \mathrm{r} /)>$ voyelles fermées $>$ voyelles moyennes $>$ voyelles ouvertes.

Les observations explicitées dans la loi des codas font l'objet de plusieurs contraintes dont la première contribue à définir $\mathrm{CV}$ comme syllabe canonique en interdisant les constituants complexes.

(20) *COMPLEXE : Aucun constituant de la syllabe n'est complexe

37 Cette contrainte doit être spécifiée pour chaque constituant car une langue peut fort bien autoriser les attaques complexes et rejeter les noyaux ou les coda doubles. On distinguera donc entre *COMPL-A (les attaques complexes ne sont pas licites) et *COMPL-C (les codas complexes ne sont pas licites).

38 La partie (b) de la loi des codas affirme qu'en position de coda, une consonne est plus faible qu'en position d'attaque ce dont témoigne l'anglais par exemple, où une occlusive est aspirée à l'initiale mais jamais dans la coda d'une syllabe. Dans les langues qui autorisent les syllabes fermées, la contrainte *CODA est largement transgressée, mais elle ne l'est pas toujours de la même manière. *CODA recouvre en fait une famille de contraintes dont (21) offre quelques exemples.

\begin{tabular}{|l|l|l|l|}
\hline$(21)$ & a) & *CODA-r : & /r/ n'est pas autorisé en position de coda \\
\hline & b) & *CODA-O : & Une occlusive n'est pas autorisée en position de coda \\
\hline & c) & *CODA-F : & Une fricative n'est pas autorisée en position de coda \\
\hline
\end{tabular}

39 La relation de dominance entre les différentes sous-divisions de (21) est donnée par l'échelle de sonorité. (21a) nous permet déjà de rendre compte de (10) et de (13), c'est-àdire de l'élision de $/ \mathrm{r} /$ soit comme premier élément d'une coda branchée comme dans tarte [ta:t] soit à la finale absolue comme dans hiver [ive:]. Cette relation de dominance explique aussi pourquoi les formes comme en (14) sont moins fréquentes.

aussi qu'à l'intérieur d'une syllabe la sonorité des éléments doit croître de la périphérie au sommet de la syllabe. Ainsi les suites /prV/, /Vnt/ respectent ce principe mais pas les configurations /stV/, ou /Vpr/. Ce principe est énoncé en (22) sous forme de contrainte.

\section{(22) SONORITE HARMONIQUE DES SEGMENTS (SHS)}

41 SHS : La sonorité des segments doit diminuer du noyau à la périphérie

(22) conjugué avec (20) crée des syllabes optimales. Si l'on accepte qu'il s'agit bien ici de consonnes de codas, la relation de dominance entre (20) et (22) doit donner la préférence à (22), ce qui donne : SHS >> *COMPL-C (SHS domine *COMPL-C), c'est-à-dire qu'une forme 
de surface peut transgresser ${ }^{*}$ COMPL-C à condition de respecter SHS. Prenons comme exemples tarte et ensemble. A, notre locuteur conservateur, simplifie les groupes OL finaux, mais généralement pas les autres groupes. Si nous imposons l'ordre proposé, SHS > *COMPL-C, en intercalant entre les deux (18) qui elle, favorise l'inertie, nous éliminons la forme [ãsãbl], mais pas [tart], ce que nous illustrons dans le tableau en (23) où une transgression de contrainte est indiquée par un astérisque '*', et une transgression rédhibitoire par '!'. La main ( $\mathbf{*})$ pointe sur le candidat optimal. Les formes de base sont identiques aux candidats (23a) et (23d), mais elles ne sont pas spécifiées afin de ne pas multiplier les tableaux.

(23) SHS >> MAXIO >> *COMPL-C

\begin{tabular}{|l|l|l|l|l|}
\hline \multicolumn{2}{|c|}{ candidats } & SHS & MAX & *COMPL-C \\
\hline \hline aa. & ensembl' & $*$ ! & & $*$ \\
\hline bb. & ensemb' & & $*$ & \\
\hline cc. & ensem'l & & $*$ & \\
\hline dd. & tart' & & & $*$ \\
\hline ee. & ta't & & $* !$ & \\
\hline ff. & tar' & & $* !$ & \\
\hline
\end{tabular}

43 La hiérarchie proposée rejette les candidats (23a, e, f) et les candidats retenus sont (23b, c, d). La forme (23c) quoique optimale ici, doit être éliminée et nous reviendrons sur son traitement. (23d) est une prononciation plus conservatrice, celle de notre locuteur A. Dans un parler moins formel, MAXIO n'intervient pas de cette façon et son rang est moins élevé dans la hiérarchie, ce que nous illustrons en (24).

(24) SHS >> *COMPL-C >> MAXIO

\begin{tabular}{|l|l|l|l|l|}
\hline \multicolumn{2}{|c|}{ candidats } & SHS & *COMPL-C & MAXIO \\
\hline aa. & ensembl' & $* !$ & $*$ & \\
\hline bb. f $^{\prime}$ & ensemb' & & & $*$ \\
\hline cc. & ensem'l & & & $*$ \\
\hline dd. & tart' & & $* !$ & \\
\hline ee. & ta't & & & $*$ \\
\hline ff. & tar' & & & $*$ \\
\hline
\end{tabular}

44 (24) indique clairement qu'une transgression de ${ }^{*}$ COMPL-C est préférable à une transgression de SHS, et en effet les groupes OL sont systématiquement simplifiés alors 
que la simplification du groupe $\mathrm{rC}$ est plus sporadique. Mais (24) n'apporte pas de véritable solution puisque quatre candidats sont optimaux alors que deux d'entre eux $(24 \mathrm{c}, \mathrm{f})$ ne sont pas attestés. Rappelons pour comparaison les autres cas de chute finale :

\begin{tabular}{|l|l|l|}
\hline$(25)$ & correct & {$[\mathrm{kor \varepsilon k}]$} \\
\hline & reste & {$[\mathrm{r \varepsilon s}]$} \\
\hline & valse & {$[\mathrm{val}]$} \\
\hline
\end{tabular}

En (25) et en (24b) la consonne finale est élidée, alors que c'est la première consonne du groupe en (24e). Ceci est dû aux effets de deux contraintes, une contrainte de fidélité (CONTIGUITE) qui comme les autres contraintes de ce groupe tend à préserver les structures de base et une famille de contraintes de marque, *CODA, définie en (21).

\begin{tabular}{|l|l|l|}
\hline$(26)$ & CONTIGUITE : & $\begin{array}{l}\text { Si deux éléments sont contigus dans la forme de base, ils le sont aussi dans } \\
\text { la forme de surface. }\end{array}$ \\
\hline
\end{tabular}

CONTIGUITE nous permet de comprendre pourquoi ce sont les segments périphériques qui sont élidés et rend compte ainsi de (25). Dans (24b), la liquide qui tombe est finale de mot et CONTIGUITE est aussi respectée, ce qui n'est pas vrai pour (24e). Nous avons aussi vu en (21) que certaines consonnes de coda étaient plus faibles que d'autres et *CODA-r domine CONTIGUITE, toutes deux étant dominées par *COMPL-C et SHS. MAXIO garde un rang très bas dans la hiérarchie et son effet ne se fait pas sentir. Les formes de base sont toujours les formes sans élision : ensembl', tart', correct.

(27) SHS >> *COMPL-C >> *CODA-r $>>$ CONTIGUITE $>>$ MAXIO

\begin{tabular}{|c|c|c|c|c|c|c|}
\hline \multicolumn{2}{|c|}{ candidats } & \multirow{2}{*}{\begin{tabular}{|l|} 
SHS \\
$* !$
\end{tabular}} & \multirow{2}{*}{$\frac{{ }^{*} \text { COMPL-C }}{*}$} & \multirow[t]{2}{*}{${ }^{*}$ CODA-r } & \multirow[t]{2}{*}{ CONTI. } & \multirow[t]{2}{*}{ MAXIC } \\
\hline aa. & ensembl' & & & & & \\
\hline bb. $=$ & ensemb' & & & & & * \\
\hline cc. & ensem'l & & & & *! & * \\
\hline dd. & tart' & & *! & & & \\
\hline ee. & tar' & & & *! & & * \\
\hline ff. $=$ & ta't & & & & * & $*$ \\
\hline gg. & correct & & *! & & & \\
\hline hh. $=$ & correc' & & & & & $*$ \\
\hline ii. & corre't & & & & *! & * \\
\hline
\end{tabular}


CONIGUIIE permet d'eliminer les deux candidats (27c) et (271). Une transgression de CONTIGUITE n'est par contre pas rédhibitoire pour (27f) car les deux autres candidats, $(27 \mathrm{~d}, \mathrm{e})$, transgressent des contraintes dont le rang est supérieur dans la hiérarchie.

Nous avons vu comment un ensemble de contraintes phonétiquement motivées est responsable de toutes les chutes de consonnes en position de coda. Un groupe OL est aussi susceptible d'être simplifié en position d'attaque ce qui met à contribution *COMPL-A plutôt que *COMPL-C. Si après est prononcé [ape] , c'est parce qu'une attaque simple est préférable à une attaque complexe, que /r/ est une consonne faible (Lyche, 1996) et que le cadien choisit d'attribuer un rôle prédominant aux contraintes de marque plutôt qu'à des contraintes de fidélité comme MAXIO et CONTIGUITE qui garantiraient l'identité aux formes de base. Encore une fois, il s'agit d'éliminer des structures complexes en faveur de la syllabe CV. Le dernier exemple d'affaiblissement que nous avons brièvement mentionné va aussi dans cette direction. Une voyelle peut être élidée à l'initiale de mot en position de hiatus et elle le sera d'autant plus facilement que les deux voyelles sont identiques : beaucoup contents ensemble [bokukõtãsãb]. L'élision de la voyelle crée une suite régulière de syllabes $\mathrm{CV}$.

\section{Conclusion}

Les affaiblissements consonantiques que nous avons étudiés sont tous attribuables à l'interaction de quelques contraintes universelles transgressables. Une comparaison d'un registre formel avec des parlers plus spontanés nous a permis de mettre en évidence la direction du changement qui se résume comme suit :

- (i) Le groupe le plus faible est le groupe OL final et ce groupe est simplifié dans tous les styles. La nature hybride de ce groupe qui en fait présente toutes les caractéristiques d'un groupe d'attaque est à l'origine de cette situation.

- (ii) La liquide $/ \mathrm{r} /$ en position de coda est le deuxième candidat à la chute.

- (iii) Une liquide de groupe OL en position d'attaque est plus forte mais peut aussi s'amuïr.

Le lien qui unit tous ces phénomènes est la création de syllabes plus optimales et principalement la création de la syllabe universelle CV. On assiste donc à un conflit entre deux forces, entre deux familles de contraintes: d'un côté, les contraintes de fidélité poussent au conservatisme alors que les contraintes de marque provoquent des changements, imposent des structures non marquées. Dans le cas d'un registre plus formel, les contraintes de fidélité l'emportent et les différences entre les formes de base et les formes de surface sont minimes. Dans un parler plus rapide, les contraintes de marque tiennent la dragée haute aux contraintes de fidélité qui sont dans l'obligation de céder le pas. OT nous permet de présenter une analyse uniforme des différents registres. Le modèle aborde la question de la variation, qu'elle soit inter- ou intra-langue, comme tout autre phénomène phonologique. La variation est motivée soit par une meilleure perception, soit par une plus grande facilité d'articulation. Elle est attribuable à des relations conflictuelles entre diverses contraintes qui sont dominantes dans certaines variétés de langue ou chez certains locuteurs, et dominées chez d'autres. Rendre compte de la variation revient tout simplement à fixer la hiérarchie des contraintes dans une variété de langue donnée. 


\section{BIBLIOGRAPHIE}

BRUN, A. (1931) Le français de Marseille. Marseille : Institut historique de Provence. [1978, Marseille: Laffitte Reprints].

CONWELL, M. (1961) Lafayette French phonology : A descriptive comparative and historical study of a Louisiana French dialect. Ph.D. Dissertation, University of Pennsylvania.

Conwell, M. \& JUILland, A. (1963) Louisiana French Grammar. Mouton : The Hague.

GADET, F. (1992) Le français populaire. Paris : Presses Universitaires de France.

GIRARD, F. \& LYCHE, C. (1998) « L'avenir du cadien ». In D. Marley, M.-A. Hintze \& G. Parker (eds.), Linguistic Identities and Policies in France and the French-speaking World. AFLS/CILT, 177-192.

KIPARSKY, P. (1988) « Phonological change ». In F. Newmeyer (ed.), Linguistic Theory: Foundations, Cambridge: Cambridge University Press, 363-415.

KIRCHNER, R.M. (1998) An effort-based approach to consonant lenition. Ph.D. Dissertation, University of California, Los Angeles. ROA-276-0898, Rutgers Optimality Archive.

LAKS, B. (1977) «Contribution empirique à l'analyse socio-différentielle de la chute des /r/ dans les groupes consonantiques finals ». Langue Française 34, 109-125.

LYCHE, C. (1996) «Schwa metathesis in Cajun French ». Folia Linguistica 24 :3, 369-393.

LYCHE, C. (1997) « Le cadien, un français ordinaire? « In B. Caron (ed.), Proceedings of the 16th International Congress of Linguists. Oxford : Pergamon, Paper No. 0104.

MONTAPANYANE, V. (1997) Acadian French. München: Lincom Europa.

PAPEN, R. A. \& ROTTET, K.J. (1997) « A structural sketch of the Cajun French spoken in Lafourche and Terrebonne parishes ». In Valdman (ed.), French and Creole in Louisiana. New York : Plenum Press, 71-108.

PASSY, P. (1891) Études sur les changements phonétiques et leurs caractères généraux. Paris: Librairie Firmin-Didot.

PRINCE, A. \& SMOLENSKY, P. (1993) Optimality Theory : Constraint interaction in generative grammar. RuCCs Technical Report \#2, Rutgers University Center for Cognitive Science, Piscataway, N.J. TINELLI, H. (1981) Creole Phonology. Janua Linguarum, Series Practica 117, The Hague: Mouton.

THUROT, C. (1883) De la prononciation française depuis le commencement du XVI ${ }^{e}$ siècle d'après le témoignage des grammairiens. Paris: Imprimerie nationale. [1966. Slatkine Reprints, Genève.]. Venneman, T. (1988) Preference Laws for the Syllable Structure and the Explanation of Sound Change. Berlin : Mouton de Gruyter.

WACHS, S. (1997) Le relâchement de la prononciation en français parlé en Ile de France : analyses linguistique et sociolinguistique par générations. Thèse de doctorat, Université de Paris X- Nanterre. 


\section{NOTES}

1. J'adopte ici la graphie cadien plutôt que cadjin ou même cajun pour m'aligner sur la pratique des linguistes louisianais.

2. Les données sur lesquelles se base cette étude proviennent en majeure partie de trois sources différentes qui représentent une vingtaine d'heures d'écoute :

- Le corpus du Centre d'Études françaises et francophones de Louisiana State University, Baton Rouge. Je remercie le Centre de m'avoir gracieusement donné accès à ces bandes.

- Enregistrements effectués par F. Girard et moi-même dans la région de Eunice et Lafayette.

- Les enregistrements du programme radiophonique 'Cajun radio storytellers'. Ces enregistrements sont commercialisés par Côte Blanche Production, Cut Off, La.

A ces données s'ajoutent aussi les transcriptions fournies par Conwell (1961).

3. La présence d'un seul locuteur représentant le registre formel est due aux difficultés pratiques rencontrées lors des interviews. De nombreux locuteurs se taisent devant un magnétophone, sont en situation de bilinguisme infériorisé et capter les différents registres d'une même personne est une tâche insurmontable. Il serait évidemment souhaitable d'étayer l'analyse d'autres enregistrements, mais les résultats obtenus sont suffisamment clairs pour nous sembler dans un premier temps fiables.

4. Dans la phrase suivante cependant, école est prononcé avec la consonne finale: dans notres écoles [dãnotzekol].

5. Nous n'avons relevé dans les enregistrements étudiés qu'un seul groupe OL en finale : il montre

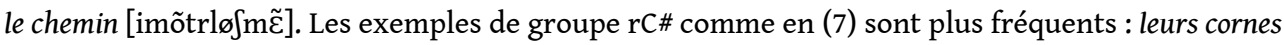
[lœrkorn].

6. La locution être après + infinitif signifie être en train de.

7. Ces exemples proviennent d'enregistrements faits par Jacques Durand et moi-même dans une petite ville de l'Hérault dans le cadre d'une large enquête sur le français du Midi.

\section{RÉSUMÉS}

Nous présentons ici un ensemble d'élisions d'éléments consonantiques en français cadien dans deux registres distincts, un registre 'formel' et un registre plus spontané. Une comparaison de ces registres nous permet de mettre en valeur les environnements les plus propices à la chute de consonnes. Nous proposons ensuite une analyse de ces phénomènes dans le cadre de la Théorie de l'Optimalité et montrons qu'ils tendent tous à favoriser la syllabe canonique CV. Dans les deux registres les contraintes de fidélité s'opposent aux contraintes de marque qui l'emportent dans le registre spontané mais qui sont dominées par les contraintes de fidélité dans un registre plus formel.

In this paper we present a number of consonant deletions in Cajun French occurring in formal speech and in casual speech. We compare the two registers in order to outline the environments which favor consonant deletion. We then propose an analysis within the framework of Optimality Theory and show that the different deletions contribute to make the canonical CV syllable optimal. We observe that in both registers faithfulness constraints conflict with 
markedness constraints which are dominant in casual speech but are lower ranked in formal speech.

\section{AUTEUR}

\section{CHANTAL LYCH}

Université d'Oslo

p.b. 1007

0315 Oslo, Norvège

chantal.lyche@matnat.uio.no 